\title{
Electrical tuning of nonlinearities in exciton-polariton condensates
}

\author{
S.I. Tsintzos, ${ }^{1, *}$ A. Tzimis,,${ }^{1,2}$ G. Stavrinidis, ${ }^{1}$ A. Trifonov, ${ }^{3}$ Z. \\ Hatzopoulos, ${ }^{1,4}$ J.J. Baumberg, ${ }^{5}$ H. Ohadi, ${ }^{5}$ and P.G. Savvidis ${ }^{1,2,3}$ \\ ${ }^{I}$ Foundation for Research and Technology-Hellas, Institute of Electronic Structure and Laser, P.O. Box 1527, 71110 Heraklion, Crete, Greece \\ ${ }^{2}$ Department of Materials Science and Technology, University of Crete, P.O. Box 2208, 71003 Heraklion, Crete, Greece \\ ${ }^{3}$ Spin Optics Laboratory, St. Petersburg State University, St. Petersburg 198504, Russia \\ ${ }^{4}$ Department of Physics, University of Crete, 71003 Heraklion, Crete, Greece \\ ${ }^{5}$ NanoPhotonics Centre, Cavendish Laboratory, University of Cambridge, Cambridge CB3 OHE, United Kingdom
}

(Dated: June 4, 2018)

\begin{abstract}
A primary limitation of the intensively-researched polaritonic systems compared to their atomic counterparts for the study of strongly correlated phenomena and many-body physics, is their relatively weak two-particle interactions compared to disorder. Here, we show how new opportunities, to enhance such on-site interactions and nonlinearities, arise by tuning the exciton-polaritons dipole moment in electrically-biased semiconductor microcavities incorporating wide quantum wells. The applied field results in a twofold enhancement of excitonexciton interactions as well as more efficiently driving relaxation towards low energy polariton states, thus reducing condensation threshold.
\end{abstract}

PACS numbers: 71.35.-y, 78.67.Pt, 71.36.+c

2018 American Institute of Physics.

Achieving the nonlinear quantum regime in photonics where the single-site effective photon interaction energy is larger than the losses, opens a plethora of interesting phenomena such as photon blockade [1], photon crystallisation [2], and opportunities to realize quantum simulators for the study of condesed matter problems such as Mott-insulator to superfluid phase transitions [3] in arrays of optical cavities. So far, the lack of scalable devices with sufficient nonlinearities and low-enough losses has been the main obstacle for the experimental realization of these phenomena. Exciton polaritons are composite quasiparticles resulting from the strong coupling of cavity photons and quantum well $(\mathrm{QW})$ excitons embedded within a microcavity (MC) [4]. Polaritons interact nonlinearly due to their excitonic component and can form macroscopically coherent condensates [5]. They are scalable to form arrays by either etching [6-8] or optical patterning [9-11] of the microcavity. However, in the presently studied systems, polariton-polariton interaction energy is smaller as compared to their line-broadening. There are two approaches towards overcoming this problem: manufacturing higher quality microcavities, or enhancing the polariton nonlinearities.

Here, we take the second approach and demonstrate twofold enhancement of the polariton-polariton interaction using wide QWs in an electrically driven MC. By exploiting the quantum confined Stark effect (QCSE) to form dipolar polaritons we demonstrate tuning of the exciton-exciton interaction. As a direct consequence of this, we obtain an enhancement of the polariton emission in both linear and lasing regimes with a simultaneous reduction of the polariton lasing threshold and shorter polariton condensate formation times due to enhanced exciton scattering. Our results are the first demonstration of the electrical tuning of nonlinearities in exciton-polariton condensates.

Exciton-exciton interactions play a key role in the strong nonlinearities present in MC polariton systems. A first at-
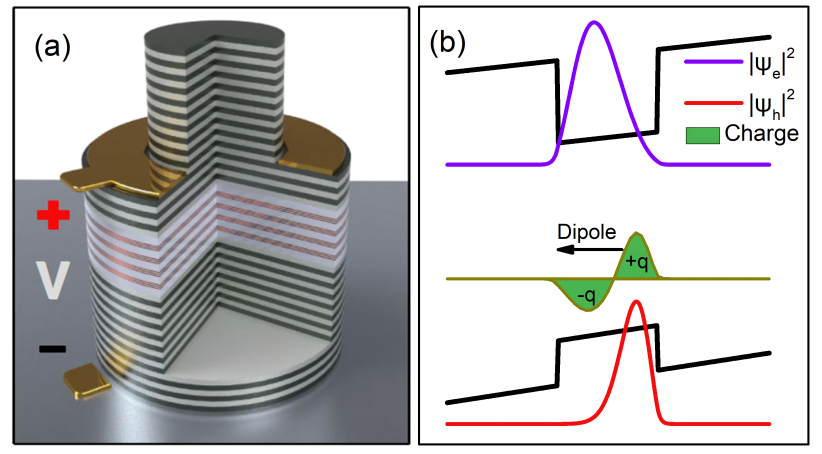

FIG. 1: (color online) (a) Schematic illustration of the electrically gated wide QW MC. (b) Schematic representation of electron and hole wavefunctions in a wide QW under the presence of electric field, and the corresponding charge distribution.

tempt to control these interactions previously suggested, was to utilise the concept of dipolaritons [12] by incorporating double asymmetric quantum wells in an electrically biased MC. Both direct (DX) and indirect (IX) excitons couple to the same cavity mode, forming a new type of polaritons with similar properties to the exciton-polariton system. Under this scheme, the coupled DX and IX share the strong oscillator strength of the direct component and the strong dipole moment of the indirect one, favoring higher polariton-polariton interactions. More recently [13], by integrating wide QWs in a simple waveguide, the formation of dipolar polaritons was observed resulting in higher interactions and therefore increased energy blueshifts when electric bias was applied. However, in both implementations only low density linear regimes are considered without examining the consequences of such enhancements on nonlinearities and polariton condensation regime.

In Fig. 1(a), the schematic of the electrically contacted MC device is illustrated. Four sets of three $18 \mathrm{~nm} / 7 \mathrm{~nm}$ 
$\mathrm{GaAs} / \mathrm{Al}_{0.3} \mathrm{Ga}_{0.7} \mathrm{As} \mathrm{QWs}$ are embedded, at the maximum of the stationary cavity field, between the top (bottom) undoped $\mathrm{AlAs} / \mathrm{Al}_{0.15} \mathrm{Ga}_{0.85} \mathrm{As} 25$ (29) distributed Bragg reflectors (DBRs). An initial etch down to the last few DBR layers forms an annular recess onto which the top contact is deposited. A second vertical etch follows for the mesa formation providing electrical isolation of the devices, while evaporation of the back contact onto the $n+$ substrate completes the processing procedure. The uniformity of the applied electric field across the active region is ensured by the high out-of-plane resistivity at the interfaces formed by alternating DBR layers.

In inorganic semiconductor MC systems, the dominating scattering mechanism contributing to the intensity of the lower polariton branch (LPB) luminescence and formation of polariton condensates via efficient relaxation towards low energy states at the bottom of LP dispersion curve is excitonpolariton scattering $[14,15]$. Therefore, the ability to achieve strong nonlinearities required for the single polariton quantum regime as well as improvements in the overall device performancel crucially depends on controlling the exciton-polariton interaction strength in such systems.

A unique opportunity to enhance such dipole excitonpolariton interactions is provided through application of an electric field across the MC device. The applied field pushes electrons and holes inside the QW in opposite directions and induces exciton dipole moment oriented along the growth axes as shown in Fig. 1(b). We quantify the effect of bias on the MC, by solving the Schrodinger equation in combination with a variational method for the excitons $[16,17]$ to extract the heavy hole $(\mathrm{HH})$ and light hole $(\mathrm{LH})$ exciton oscillator strengths and thus radiative times [Fig. 2(a)], dipole moment [Fig 2(a)] and excitonic Bohr radius and the exciton interaction strength constant $g_{x}$ [18] [Fig. 2(b)] as a function of electric field. Increase in exciton radiative time with electric field arises from the decrease in electron and hole wavefunction overlap due to the QCSE [Fig. 2(a)]. Simultaneously, application of electric field provides control over the excitonexciton interaction $g_{x}$, which arises from i) exchange interactions $\left(g_{\text {exc }}\right)$ and ii) dipole-dipole interactions $\left(g_{d i p}\right)$. Both contributions are shown in Fig. 2(b) as a function of electric field. The former depends on exciton Bohr radius [19] while the latter is enhanced with the increase of the effective dipole length shown in [13].

We initially employ streak camera measurements at $20 \mathrm{~K}$ to track the relaxation dynamics in both linear and nonlinear regimes. Notably, since time-resolved photoluminescence (TRPL) experiments measure directly relaxation and scattering dynamics, exciton-polariton interaction strengths which control these processes can be experimentally assessed during the application of electrical bias. In Fig. 3(a) TRPL is recorded for varying electrical bias in the linear low-density excitation regime. For all traces in addition to photoluminescence (PL), we allow a small fraction of pump laser to be collected marking the arrival of the excitation pulse at $t=0 \mathrm{ps}$. Following nonresonant excitation the PL reaches a maximum at $160 \mathrm{ps}$ and decays over hundreds of ps. We attribute the
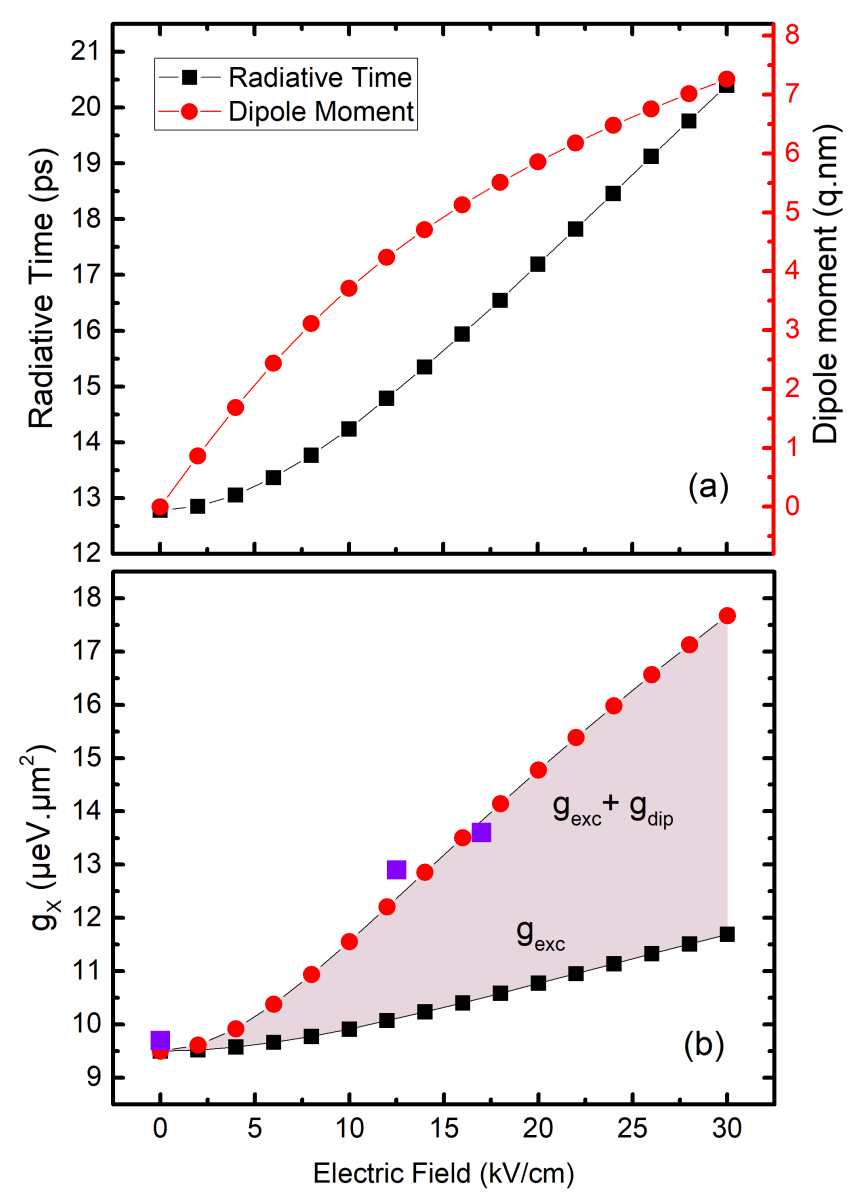

FIG. 2: (color online) Theoretical calculations as a function of the electric field for (a) the radiative lifetime and dipole moment for the $\mathrm{HH}$ excitons, (b) HH exciton interaction strength. The three purple square points are experimental data exported as discussed in the text.

increase in the integrated PL intensity to enhanced scattering and relaxation of polaritons on the LPB caused by the applied electrical bias. The PL intensity reaches maximum at an applied field of $17 \mathrm{kV} / \mathrm{cm}$, above which LO phonon assisted tunnelling between adjacent QWs leads to significant loss in the PL intensity and strong charging of the QWs [20].

Similarly, strong PL intensity enhancements in the presence of electric field is also achieved at higher excitation powers in the polariton lasing regime. Due to the presence of strong nonlinearities and stimulation effects affecting relaxation, the maximum PL intensity and condensation is shifted to earlier times $\sim 90 \mathrm{ps}$. The sharp rise in the PL intensity at $50 \mathrm{ps}$ marks the time when the system reaches occupancy of one where polariton condensation then dominates the dynamics.

To interpret our experimental results and link PL intensity enhancements to changes in the scattering rates driven by the exciton-polariton interaction strength in both linear and nonlinear regimes we developed a simplified rate equations model based on the Boltzmann kinetic equations [15], neglecting the momentum-dependence of the various scattering processes. Following the example of [21] we split the reservoir in two 


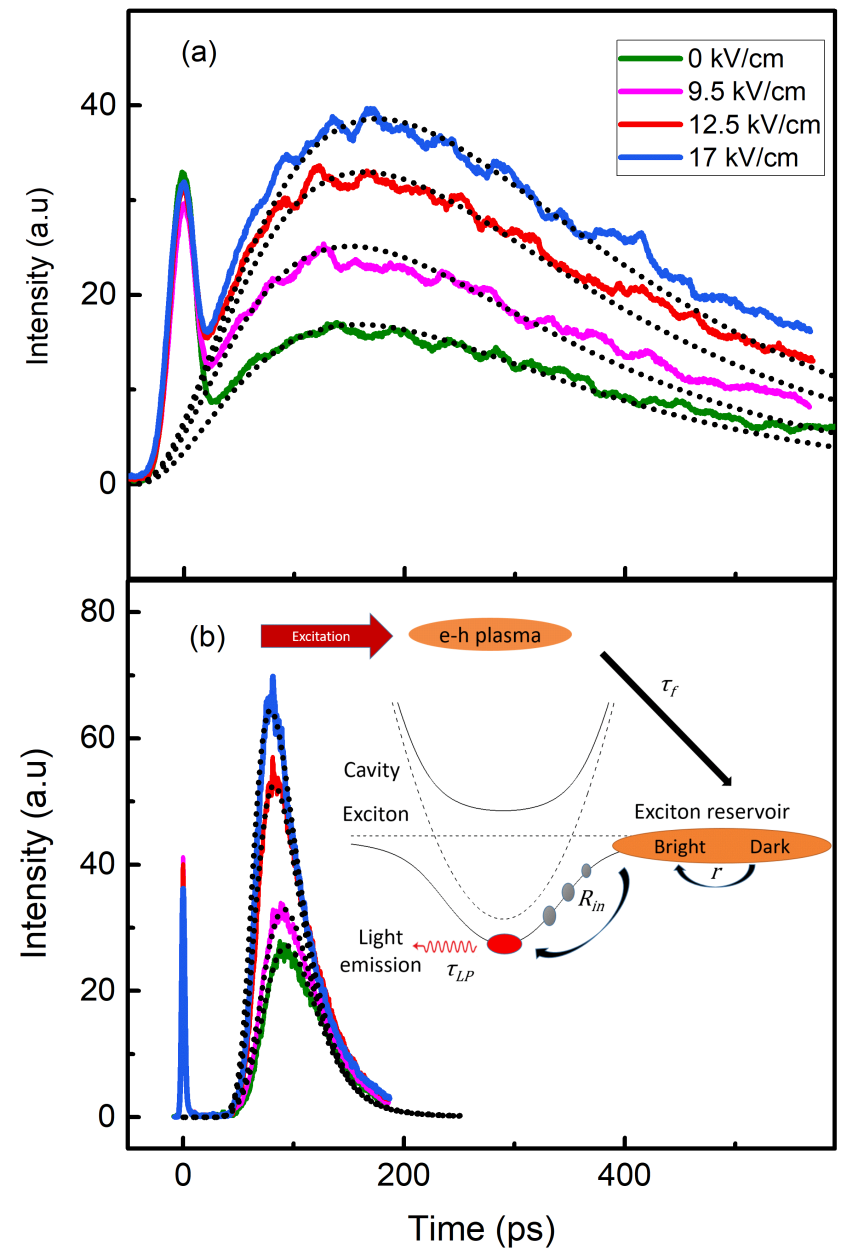

FIG. 3: (color online) Time-resolved PL at four discrete electric field values for (a) below laser threshold $(P=0.2 \mathrm{~mW})$ and (b) above laser threshold $(P=4.25 \mathrm{~mW})$. The inset of (b) is a schematic illustration of the various relaxation mechanisms incorporated in the rate equations.

parts, labelled as dark and bright respectively [inset graph in Fig. 3(b)]. The bright excitonic reservoir describes the excitons, with a density $n_{B X}$ that conserve energy and momentum for scattering into the lower polariton branch. The dark excitons, $n_{D X}$, which are out of the light cone, cannot scatter directly into the condensate, but simply feed the bright subset of excitons with a rate denoted as $r$ through inelastic scattering. To improve the validity of our model, we take into account exciton formation, the radiative and non-radiative exciton losses while the exciton-polariton scattering is considered to be the main process of polariton relaxation to the bottom of the LPB. The detailed equations are shown below while the exact values of the parameters used to fit the experimental data are given in Table 1 in the supplementary material.

$$
\frac{d n_{e h}(t)}{d t}=P-\frac{n_{e h}(t)}{\tau_{e h}}-\frac{n_{e h}(t)}{\tau_{f}}
$$

$$
\begin{gathered}
\frac{d n_{D X}(t)}{d t}=\frac{n_{e h}(t)}{\tau_{f}}-\frac{n_{D X}(t)}{\tau_{n r}} \\
-r\left[\frac{n_{D X}(t)}{k}-n_{B X}(t)\right] \\
\frac{d n_{B X}(t)}{d t}=r\left[\frac{n_{D X}(t)}{k}-n_{B X}(t)\right]-\frac{n_{B X}(t)}{\tau_{\text {rad }}} \\
-R_{\text {in }} n_{B X}^{2}(t)\left[N_{L P}(t)+1\right]+R_{\text {out }} n_{B X} N_{L P}(t) \\
\frac{N_{L P}(t)}{d t}=R_{\text {in }} n_{B X}^{2}(t)\left[N_{L P}(t)+1\right]-R_{\text {out }} n_{B X} N_{L P}(t) \\
-\frac{N_{L P}(t)}{\tau_{L P}}
\end{gathered}
$$

Here, $k$ is the equilibration rate of the densities between the two reservoirs, $n_{e h}$ is the density of free carriers, while $N_{L P}$ is the population of the polariton ground state. The time constants $\tau_{e h}$ and $\tau_{f}$ refer to the free carrier lifetime and the exciton formation time, $\tau_{n r}$ the non-radiave lifetime of the dark excitons, $\tau_{\text {rad }}$ the radiative lifetime of the bright excitons, $R_{\text {in,out }}$ are the scattering rates into and out the LPB to the bright exciton reservoir and $\tau_{L P}$ is the polariton lifetime. We approximate the inscattering and outscaterring rates as $R_{\text {in }}=\gamma \exp (-\varepsilon / k T)$ and $R_{\text {out }}=R_{\text {in }} \exp (-\varepsilon / k T)$ [22], where $\varepsilon=-10 \mathrm{meV}$ is the energy difference between the $\mathrm{HH}$ exciton and the minimum of the LPB at $14 \mathrm{~K}$ under zero external field, while $\gamma$ is a parameter which quantifies the electric field dependence of the inscattering rate. Furthermore, we account for both the exciton redshift and the reduction of oscillator strength changes related to application of the external electric field which affects both Hopfield coefficients and polariton lifetimes $\tau_{L P}$ using three harmonic oscillator model.

Excellent agreement between the measured and the modelled PL intensity traces (dotted lines) can be seen in Fig. 3(a) and 3(b). In the present model, only one fitting parameter changes with electric field, namely $\gamma$ which quantifies the field dependent polariton relaxation rate. The observed PL enhancement, in both linear and non-linear regimes, arises from: a) the increase in exciton radiative lifetime, with increasing electric field, which suppresses PL emission near the relaxation bottleneck and enhances PL emission near $k_{\|}=0$ and b) the increase in the exciton-exciton interaction strength which enhances relaxation towards $k_{\|}=0$ polariton states. Incorporating previously-calculated theoretical values for the exciton radiative lifetime, keeping the remaining parameters constant (including exciton scattering rate), the model predicts only weak enhancement in the PL intensity resulting from any reduction in bright exciton reservoir radiative losses, which is not sufficient to fit our experimental data. Clearly, at high electric fields the second term related to exciton-polariton scattering dominates and contributes strongly to the PL increase. Taking this into account, experimental data both in the linear and nonlinear regimes at electric fields of 12.5 and $17 \mathrm{kV} / \mathrm{cm}$ can be fit using higher $\gamma$ parameter values of 105 $\mathrm{um}^{4} \mathrm{ps}^{-1}$ and $150 \mathrm{um}^{4} \mathrm{ps}^{-1}$ compared to zero bias scattering time of $56 \mathrm{um}^{4} \mathrm{ps}^{-1}$ (see also Table 1 in the supplementary 

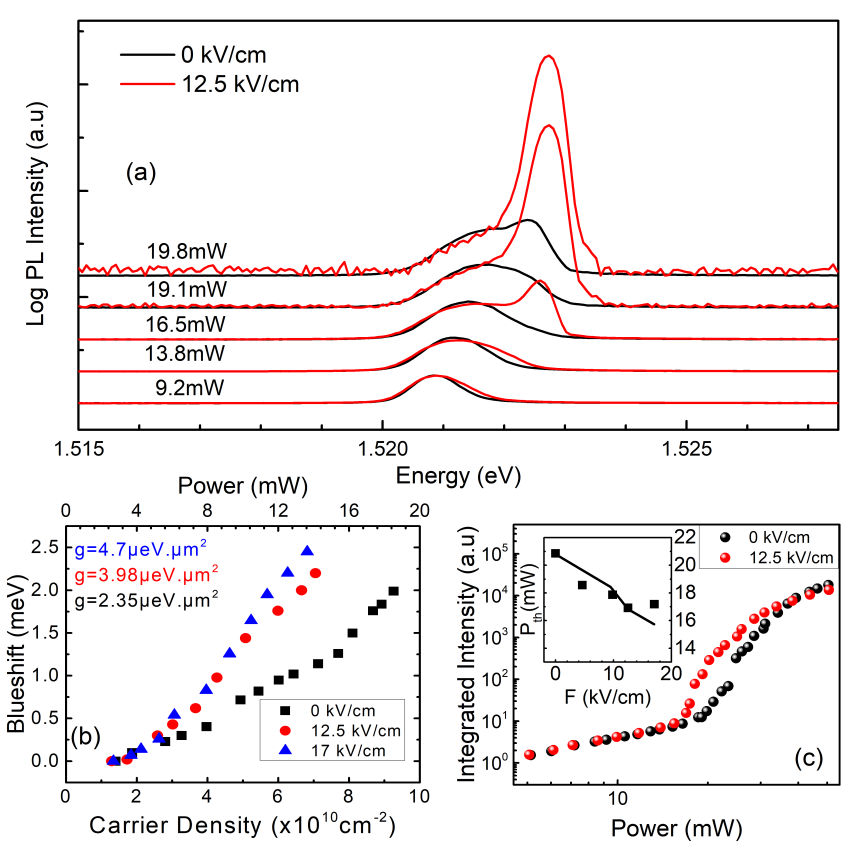

FIG. 4: (color online) (a) Logarithmic power dependent PL spectra at zero (black) and $12.5 \mathrm{kV} / \mathrm{cm}$ (red) electric fields. (b) The polariton energy blueshift at three different electric field values. (c) Integrated PL emission extracted from the corresponding spectra of figure (a). Reduction of polariton laser threshold versus electric field appears in the inset figure. The squares are experimental data while the solid line is obtained from theoretical calculations.

material). This corresponds to enhancements of the inscattering rate $R_{i n}$ by a factor of $87 \%$ and $267 \%$ respectively. Since the exciton-polariton scattering rate is proportional to $X_{L P} g_{x}^{2}$, where $X_{L P}$ is the excitonic fraction at the LPB [23], the experimentally obtained scattering rate enhancements can be compared with the corresponding electric field induced changes in the exciton interaction constant $g_{x}$. Using theoretical values for $g_{x}$ shown in Fig 2(d) the calculated field induced changes in the exciton-polariton scattering rate are estimated as $78 \%$ and $258 \%$ and are in excellent quantitative agreement with the experiment.

Independent estimates of exciton-exciton interaction strength $g_{x}$ can be obtained performing $\mathrm{cw}$ power dependent measurements of the LPB energy blueshifts while varying electrical bias. Assuming that the LPB blueshift depends on the exciton fraction in the LPB $k_{\|}=0$ state, we select a device with a smaller exciton-photon detuning(-4.5meV) which permits more precise measurement of $g_{x}$. Two indicative sets of PL data, recorded from the LPB zero k-state at 20K, at the same excitation power for zero and $12.5 \mathrm{kV} / \mathrm{cm}$ electric field values are plotted in Fig. 4(a), while the extracted blueshift energy is shown in Fig. 4(b). As expected, the gradual energy blueshift slope changes with the increasing electric field, providing evidence for enhanced dipole-dipole interactions. Using the experimentally obtained dark reservoir carrier lifetimes, we calibrate the horizontal axis to obtain the steady state carrier density $N$ and estimate the exciton-polariton interaction constant $g_{x p}$ by fitting the relation $\Delta E=g_{x p} \cdot N$ to the linear part of the data shown in Fig. 4(c). This yields $g_{x p}=2.35 \mu \mathrm{eV} \mu \mathrm{m}^{2}$ at $\mathrm{F}=0 \mathrm{kV} / \mathrm{cm}, g_{x p}=3.98 \mu \mathrm{eV} \mu \mathrm{m}^{2}$ at $\mathrm{F}=12.5 \mathrm{kV} / \mathrm{cm}$ and $g_{x p}=4.7 \mu \mathrm{eV} \mu \mathrm{m}^{2}$ at $\mathrm{F}=17 \mathrm{kV} / \mathrm{cm}$.

To obtain exciton-exciton interaction strengths from the corresponding exciton-polariton total, the latter has to be normalized by field-dependent exciton Hopfield coefficients [24]. For the electric field values above, after correcting for the exciton fraction we find $g_{x}=9.85 \mu \mathrm{eV} \mu \mathrm{m}^{2}(0 \mathrm{kV} / \mathrm{cm})$, $g_{x p}=12.98 \mu \mathrm{eV} \mu \mathrm{m}^{2}(12.5 \mathrm{kV} / \mathrm{cm})$ and $g_{x p}=13.6 \mu \mathrm{eV} \mu \mathrm{m}^{2}$ $(17 \mathrm{kV} / \mathrm{cm})$. These experimental values are in agreement with the theoretical ones presented in the Fig. 2(d). Moreover, direct calculation of the exciton-polariton scattering rate using the above extracted $g_{x}$ values reveals an enhancement of $83 \%$ $(12.5 \mathrm{kV} / \mathrm{cm})$ and $250 \%(17 \mathrm{kV} / \mathrm{cm})$ respectively, which are in agreement with the values found through the rate equation model.

The integrated PL emission extracted from the spectra in Fig. 4(a), is shown in Fig. 4(c). The field-dependent excitonpolariton scattering rate gradually lowers the polariton lasing threshold as shown in the inset of Fig. 4(a) where experimental measurements are shown by squares while the solid line is obtained from the steady state solution of the rate equations by fixing the polariton occupation number $N_{L P}=1$. Polariton lasing threshold reduction up to $20 \%$ is achieved at the maximum applied electric field of $17 \mathrm{kV} / \mathrm{cm}$.

In conclusion, we demonstrate electrical manipulation of the exciton-exciton interaction strength by applying external electric field to a wide-QW microcavity. Equivalent results are obtained for the exciton-exciton interaction constant using time-resolved PL and CW power dependent blueshift measurements. We construct theoretical model which is in good agreement and interprets our experimental findings. All the above corroborate the possibility of manipulating polaritonpolariton interactions through external electrical fields. Furthermore, with careful band-structure design and device optimization, allowing application of stronger electric fields, it will be possible to obtain even higher $g_{x}$ values. Such controlled enhancement of exciton interactions, may prove crucial for realization of non classical light sources relying on polariton quantum blockade.

ST acknowledges the financial support of the Stavros Niarchos Foundation within the framework of the project ARCHERS, PS acknowledges bilateral Greece-Russia Polisimulator project co-financed by Greece and the EU Regional Development Fund, AT acknowledges the RussianGreek project supported by Ministry of Education and Science of The Russian Federation (project RFMEFI61617X0085).

Electronic address: simostsiephysics.uoc.gr

[1] K.M. Birnbaum, A. Boca, R. Miller, A.D. Boozer, T E. Northup, and H.J. Kimble, Nature 436, 87-90 (2005).

[2] D.E. Chang, V. Gritsev, G. Morigi, V. Vuletic, M.D. Lukin, and E.A. Demler, Nature Physics 4, 884-889 (2008). 
[3] M. Greiner, O. Mandel, T. Esslinger, T.W. Hnsch, and I. Bloch, Nature, 415, 39 (2002).

[4] C. Weisbuch,M. Nishioka, A. Ishikawa, and Y., Arakawa, Phys. Rev. Lett, 69, 3314 (1992).

[5] J. Kasprzak, M. Richard, S. Kundermann, A. Baas, P.Jeambrun, J.M. Keeling, F.M. Marchetti, M.H.Szymanska, R. Andre, J.L. Staehli, V. Savona, P.B.Littlewood, B. Deveaud, and Le S. Dang, Nature, 443, 409 (2006).

[6] E. Wertz, L. Ferrier, D.D. Solnyshkov, R. Johne, D. Sanvitto, A. Lematre, I. Sagnes, R. Grousson, A. V.Kavokin, P. Senellart, G. Malpuech, and J. Bloch, Nature Physics, 6, 860 (2010).

[7] T. Gao, P.S. Eldridge, T.C.H. Liew, S.I. Tsintzos, G.Stavrinidis, G. Deligeorgis, Z. Hatzopoulos, and P.G. Savvidis, Phys. Rev. B 85, 235102 (2012).

[8] L. Ferrier, E. Wertz, R. Johne, D.D. Solnyshkov, P. Senellart, I. Sagnes, A. Lemaitre, G. Malpuech, and J. Bloch, Phys. Rev. Lett. 106, 126401 (2011)

[9] G. Tosi, G. Christmann, N.G. Berloff, P. Tsotsis, T. Gao, Z. Hatzopoulos, P.G. Savvidis, and J.J. Baumberg, Nat. Phys. 8, 190 (2012).

[10] A. Askitopoulos, K. Kalinin, T.C.H. Liew, P. Cillibrizzi, Z. Hatzopoulos, P.G. Savvidis, N.G. Berloff, and P.G. Lagoudakis, Phys. Rev. B 93, 205307 (2016).

[11] H. Ohadi, A.J. Ramsay, H.Sigurdssom, Y.del Valle-Inclan Redondo, S.I. Tsintzos, Z. Hatzopoulos, T.C.H. Liew, I.A Shelykh, Y.G. Rubo, P.G. Savvidis, and J.J. Baumberg, Phys. Rev. Lett. 119, 067401 (2016).

[12] P. Cristofolini, G. Christmann, S.I. Tsintzos, G. Deligeorgis, G. Konstantinidis, Z. Hatzopoulos, P.G. Savvidis, and J.J.Baumberg, Science 336, 704 (2012).
[13] I. Rosenberg, Y. Mazuz-Harpaz, and R. Rapaport, Phys. Rev. B 93, $195151(2016)$

[14] G. Malpuech, A. Kavokin, A. Di Carlo, J.J. Baumberg, Phys. Rev. B 65, 153310 (2002)

[15] D. Porras, C. Ciuti, J.J. Baumberg,and C. Tejedor, Phys. Rev. B 66, 085304 (2002)

[16] P. Harrison, Quantum Wells Wires and Dots, Willey, (2005).

[17] E.S. Khramtsov, P.A. Belov, P.S. Grigoryev, I.V. Ignatiev, S.Yu. Verbin, Yu.P. Efimov, S.A. Eliseev, V.A. Lovtcius, V.V. Petrov, and S.L. Yakovlev, J.App.Phys. 119, 184301 (2016).

[18] C. Ciuti, V. Savona, C. Piermarocchi, and A. Quattropani, Phys. Rev. B 58, 7926 (1998).

[19] T. Byrnes, G.V. Kolmakov, R.Ya. Kezerashvili, and Y. Yamamoto, Phys. Rev. B 90, 125314 (2014).

[20] P. Tsotsis, S.I. Tsintzos, G. Christmann, P.G. Lagoudakis, S. Kyrienko, I.A. Shelykh, J.J. Baumberg, A.V. Kavokin, Z. Hatzopoulos, P.S. Eldridge and P. G. Savvidis, Phys. Rev. Applied 2, 014002 (2014)

[21] G. Nardim, K.G. Lagoudakis, M. Wouters, M. Richard, A. Baas, R. Andre, Le Si Dang, B. Pietka,and B. DeveaudPledran,Phys. Rev. Lett. 103, 256402 (2009).

[22] M. Wouters, V. Savona, Phys. Rev. B 79, 165302 (2009)

[23] F. Tassone, Y. Yamamoto, Phys. Rev. B 59, 10830 (1999)

[24] R. Hahe, C. Brimont, P. Valvin, T. Guillet, F. Li, M. Leroux, J. Zuniga-Perez, X. Lafosse, G. Patriarche, and S. Bouchoule Phys. Rev. B 92, 235308 (2015).

[25] A. Kavokin, J.J. Baumberg, G. Malpuech,and F.P. Laussy, Microvavities, Oxford University Press (2007) 